


\section{Ciencias humanas y objetivación: reflexiones en torno a la crítica política del saber elaborada por Michel Foucault}

\section{Human Sciences and objectification: reflections about the political critique of knowledge made by Michel Foucault}

Iván Gabriel Dalmau

Universidad Nacional de San Martín, Argentina

ivandalmau@yahoo.com.ar

Resumen: El propósito de este trabajo es llevar a cabo una lectura minuciosa respecto de la relación entre la problematización foucaulteana del saber y su propuesta de llevar a cabo una crítica política de las ciencias humanas. Por lo tanto, pondremos el foco de nuestra lectura en diferentes libros, lecciones y artículos que el filósofo francés le dedica a dicha cuestión.

Palabras clave: Foucault, Crítica, Saber, Genealogía, Ciencias Humanas.

Abstract: The aim of this work is to make a close reading of the relationship between the Foucauldean problematization of knowledge and his proposal of work out a political critique of human sciences. Hence, we will focus our reading in different books, lessons and articles that the French philosopher wrote about this topic.

Keywords: Foucault, Critique, Knowledge, Genealogy, Human Sciences.

Recibido: 27 de diciembre de 2018

Aceptado: 13 de abril de 2019 http://dx.doi.org/10.15174/rv.v0i24.437 


\section{A modo de introducción}

Foucault, en un procedimiento que le era propio, no ha cesado hasta el final de su vida de "releer", de resituar y de reinterpretar sus antiguos trabajos a la luz de los últimos, en una suerte de reactualización incesante.

FontANA y BERTANi (1997: 248)

$\mathrm{F}^{\mathrm{n} \text { el presente artículo se pretende llevar a cabo una lectura }}$ caulteana, para rastrear qué tipo de crítica de las ciencias humanas permite llevar a cabo la crítica política del saber propuesta por el filósofo. Cabe remarcar que en nuestro abordaje del archivo no nos ocuparemos de analizar las referencias de Michel Foucault a otros filósofos o corrientes filosóficas en términos de su plausibilidad filológica e histórico-conceptual, sino que más bien nos valdremos de las mismas en función del modo en que se inscriben en la economía del discurso foucaulteano. Por lo tanto, si bien nos apoyaremos en la manera en que el pensador francés caracteriza los senderos que se abren en torno al legado kantiano y, también, de las contraposiciones entre arqueología del saber y teoría del conocimiento, y entre crítica política del saber y crítica de la ideología, no evaluaremos lo ajustado del modo en que Foucault caracteriza las nociones frente a las que distingue las herramientas que forja, sino que más bien nos apoyaremos en dichos juegos de oposiciones para dar cuenta del tipo de crítica de los saberes acerca de "lo humano" que las herramientas foucaulteanas posibilitan practicar.

En lo que a la estrategia argumental respecta, dividiremos las líneas que se despliegan a continuación en tres apartados y otro con la reflexión final. Apoyados en el característico gesto foucaulteano de recuperación y reelaboración de sus trabajos, señalado por Ales- 
sandro Fontana y Mauro Bertani en la cita que hemos colocado como epígrafe y remarcado de manera recurrente por las y los especialistas (Wallenstein, 2013: 10; Lehm /Vatter, 2014: 1-2; Raffin, 2015: 58-63), en el primer apartado nos detendremos en el abordaje recurrente de las ciencias humanas elaborado por Michel Foucault dentro del marco de problematización de la filosofía como actividad de diagnóstico de la actualidad. Tras lo cual, revisaremos la caracterización foucaulteana de la noción de saber, tal como la perfilara en su clásico trabajo de finales de los años sesenta (Foucault, 1969). Luego, pondremos el foco en lo que denominamos como ecos arqueológicos de la genealogía, para de tal modo poder dar cuenta de la densidad epistemológica y ontológico-política que articula la propuesta foucaulteana de llevar a cabo una crítica política del saber (Foucault, 2004a).

Punto de partida: la problematización de las ciencias humanas en el seno del ejercicio de la filosofía como actividad de diagnóstico

A propósito de Nietzsche, podemos volver sobre su pregunta: para él, el filósofo es quien diagnostica el estado del pensamiento. Por cierto, se pueden concebir dos clases de flósofos, el que abre nuevos caminos al pensamiento, como Heidegger, y el que juega en cierta forma el rol de arqueólogo, que estudia el espacio en el que se despliega el pensamiento, asi como sus condiciones, su modo de constitución

Foucault (1994a: 553)

En la primera clase del curso dictado en el Collège de France durante el ciclo lectivo 1982-1983, es decir la clase del 5 de Enero 
de 1983 con la que diera inicio al curso Le gouvernement de soi et des autres, Foucault se vale de una presentación del modo en que Immanuel Kant respondió a la pregunta "Was ist Aufklärung?" para inscribir su propia labor en una modulación de la crítica:

Me parece que la elección filosófica a la que nos encontramos confrontados actualmente es ésta. Hay que optar por una filosofía crítica que se presentará como una filosofía analítica de la verdad en general, o por un pensamiento crítico que tomará la forma de una ontología de nosotros mismos, de una ontología de la actualidad. Y es esta forma de filosofía la que, de Hegel a la Escuela de Frankfurt, pasando por Nietzsche, Max Weber, etc., ha fundado una forma de reflexión a la cual, desde luego, me vinculo en la medida en que puedo (Foucault, 2008: 22).

Consideramos fundamental detenernos en que, frente a la realización de una "analítica de la verdad en general" - preocupada por las posibilidades del conocimiento y sus límites infranqueables-, Foucault reivindica la práctica de la crítica como una ontología de la actualidad. De lo que se trata, entonces, es de una inflexión del gesto crítico; que, en lugar de erigirse como una "analítica de la verdad", se enmarca en el proyecto de dar cuenta de la constitución histórica de focos de experiencia, para poder desentrañar ontológico-políticamente la actualidad y problematizar la contingencia y las posibilidades de franqueamiento de lo presuntamente "universal y necesario" (Mascaretti, 2014: 139).

Retomando el gesto foucaulteano de recuperación y reelaboración recurrente de sus trabajos -al que hemos aludido en la introducción- consideramos pertinente remitirnos al modo en que el filósofo elabora las nociones de saber y genealogía, desde la perspectiva de la manera en que problematiza el carácter crítico de la práctica filosófica. Por lo tanto, dirigiremos nuestra lectura hacia la 
problematización arqueológica del saber, tras lo cual nos detendremos en los que podrían denominarse como "ecos arqueológicos de la genealogía". Sin embargo, antes de proseguir nuestra lectura, para disipar cualquier sospecha de "forzamiento teleológico" de la interpretación propuesta, quisiéramos remarcar una serie de cuestiones. ${ }^{1}$

En primer lugar, al referirse a Nietzsche -en la cita que hemos colocado como epígrafe- y al estructuralismo (Foucault, 1994b), referencias introducidas en el marco de entrevistas que tuvieron lugar a mediados de la década del sesenta en el contexto de la publicación de Les mots et les choses (Foucault, 1966), Foucault vinculó explícitamente la actividad filosófica con el diagnóstico de la actualidad. Por otra parte, no puede desconocerse que en las arqueologías acerca de la locura (Foucault, 1972)² y la clínica (Foucault, 1963), el filósofo problematiza la imbricación entre la formación de determinados saberes y las transformaciones en las prácticas institucionales, en sintonía con sus posteriores indagaciones genealógicas desarrolladas en los años setenta. Relación que parece elidida en Les mots et les choses, cuya preocupación ontológico-política resulta insoslayable, puesto que de lo que allí se trata es de problematizar la constitución del "hombre", de modo tal que pueda desasirse de la grilla de inteligibilidad forjada por el humanismo que hace de dicha figura el punto de partida tanto de la reflexión teórico-epistemológica como de la indagación prácticopolítica (Paltrinieri, 2014: 108).

$\mathrm{Al}$ respecto, no puede desconocerse que hacia el final de $L$ 'archéologie du savoir (Foucault, 1969), el filósofo explicita la posibilidad de llevar a cabo "otras arqueologías" acerca de la pintura,

${ }^{1}$ Sobre este punto, no puedo dejar de mencionar las discusiones sostenidas con los filósofos Claudio Cormick (CONICET-Uba) y Mario Gómez Pedrido (UBA). Valga esta nota al pie como forma de agradecimiento.

${ }^{2}$ Si bien remitimos a la segunda edición, para evitar confusiones recordamos que la primera fue presentada como tesis doctoral en 1961. 
la sexualidad y la política, blancos en los que se detendría a lo largo de los cursos dictados en el Collège de France (Castro, 2011: 4345). Justamente, será en el marco de sus indagaciones acerca del surgimiento del modo disciplinario de ejercicio del poder (1975), del dispositivo de sexualidad (1976), de la biopolítica (1997) y de las formas modernas de gubernamentalidad (2004a; 2004b) que Foucault se ocupará de problematizar de manera recurrente la formación del discurso de las ciencias humanas; tal como lo hiciera, por ejemplo, al reformular la mutación epistemológica abordada en Les mots et les choses (1966) en el marco del curso Sécuirté, Territoire, Popultaion (2004b), dictado luego de doce ańos de la publicación del libro (Castro-Gómez, 2010: 16). Por otra parte, querríamos remarcar que si bien por ciencias humanas Foucault alude a los estudios literarios, la sociología y las ciencias con radical "psi", en la medida en que sostiene que las mismas se configuraron a partir del plexo formado por las ciencias respecto de la vida, el trabajo y el lenguaje, para simplificar la redacción nos valemos de la noción de "ciencias humanas" en sentido amplio y englobante para aludir a la disposición epistémica en la que dichas ciencias se formaron (Foucault, 1966; 2004b).

\section{La problematización arqueológica de los saberes acerca de "lo humano"}

No se trata ni de exponer lo que los cientificos o los sabios han dicho o pensado o creído demostrar ni de reconstruir desde un punto de vista verdadero o de la ciencia normal, desde el punto de vista contemporáneo, lo que se ha dicho o pensado o demostrado, considerando el presente de una ciencia como normativo respecto de su pasado. [...] La arqueología del saber procura establecer a partir de qué disposición histórica han sido posibles las ciencias, sin identificar tal disposición 
ni con el valor racional del conocimiento ni con sus formas objetivas

En el presente apartado pondremos el foco de miras en las herramientas de trabajo arqueológico sobre los discursos tal como Foucault las sistematizara en $L$ 'archéologie du savoir (1969). Tomamos dicho libro como punto de partida en tanto que, como se explicita en la propia letra foucaulteana, puede ser leído como un ejercicio de corrección y recuperación crítica de los desarrollos desplegados a lo largo de sus investigaciones precedentes. Allí, respecto del análisis arqueológico de los discursos, el filósofo planteó que:

Pero de lo que aquí se trata, no es de neutralizar el discurso, de hacerlo el signo de otra cosa y de atravesar su espesor para alcanzar aquello que permanece silenciosamente más allá de él sino, al contrario, de mantenerlo en su consistencia, de hacerlo surgir en la complejidad que le es propia [...]. Sustituir el tesoro enigmático de las "cosas" anteriores al discurso, por la formación regular de los objetos que no se perfilan más que en él. Definir esos objetos sin referencia al fondo de las cosas, sino en relación al conjunto de las reglas que permiten formarlos como objetos de un discurso y constituyen así sus condiciones de aparición histórica (Foucault, 1969: 65).

Foucault caracteriza, entonces, el abordaje arqueológico como un trabajo de archivo realizado sobre los discursos, que consistirá en abordarlos en tanto prácticas discursivas y se orientará hacia el dar cuenta de sus condiciones de posibilidad; logrando así el establecimiento de los modos históricos de constitución de ciertas positividades, en lugar de tomarlas de antemano como evidencia y punto de partida (Muhle, 2012: 190). Forma de problematiza- 
ción en torno a la que el filósofo introdujo la noción de formación discursiva:

En el caso en que se pudiera describir, entre un cierto número de enunciados, un semejante sistema de dispersión, en el caso en que entre los objetos, los tipos de enunciación, los conceptos, las elecciones temáticas, se pudiera definir una regularidad (un orden, correlaciones, posiciones y funcionamientos, transformaciones), se dirá, por convención, que se trata de una formación discursiva [...]. Se llamarán reglas de formación a las condiciones a las cuales están sometidos los elementos de esta repartición (objetos, modalidad de enunciación, conceptos, elecciones temáticas) (Foucault, 1969: 53).

El filósofo dedicará la primera parte del libro en cuestión a desbrozar con mayor detenimiento cada uno de los aspectos que configura la citada definición, apoyándose en los discursos que había abordado en sus trabajos precedentes y reformulando la lectura de los mismos desde la perspectiva del "pasaje en limpio" de la metodología arqueológica y sus supuestos epistemológicos. Cabe destacar que el pasaje de la problematización de los objetos y las modalidades enunciativas hacia sus reglas de formación resulta un aporte epistemológico fundamental, puesto que permite desanclar la reflexión epistemológica del interior de la relación sujeto-objeto, y visibilizar los términos de la relación cognoscitiva como inmanentes a dichas reglas de formación. El registro arqueológico de problematización habilita a poner el foco de indagación en la pregunta acerca de cómo, en torno a la formación de cierto saber, se constituyeron objetos y posiciones de sujeto. Pregunta en torno a la que el discurso de las ciencias humanas es susceptible de ser analizado en tanto conjunto de prácticas que se encuentran atravesadas por reglas que de manera inmanente regulan el ejercicio 
de la función enunciativa. Dicho conjunto transformable de reglas permite dar cuenta de la regularidad que rige la dispersión de los enunciados efectivamente producidos (Brossat, 2013: 2-3).

Puede decirse, entonces, que la arqueología permite aproximarse a los discursos en tanto prácticas que forman regularmente los objetos que sólo en ella tienen lugar, al tiempo que posibilitan la constitución correlativa de formas de subjetividad. De hecho, no puede pasarse por alto que este modo de problematización de los saberes en tanto prácticas discursivas permitió a Foucault plantear la oposición entre la teoría del conocimiento, que se ocuparía de dar cuenta de la relación sujeto-objeto, y la arqueología del saber que se coloca por fuera de dicha relación; además de que procura enfocarse en la formación de ambos términos en tanto inmanentes al ejercicio de la función enunciativa (Foucault, 1994c: 730). En estrecha vinculación con lo antedicho debe ser revisada la caracterización de la arqueología de las ciencias humanas desarrollada en Les mots et los choses; en el prefacio de dicho libro, el filósofo remarcó que la reflexión epistemológica puesta en práctica arqueológicamente prescinde de todo cariz teleológico y, por lo tanto, se ocupa de problematizar la constitución de los saberes en su historicidad al remitirlos a la episteme en cuyo seno pudieron formarse (Foucault, 1966: 13). Caracterización del trabajo arqueológico que Foucault complementa en el último capítulo de L'archéologie du savoir, titulado "Science et savoir", en el que contrapone explícitamente el abordaje arqueológico frente a la realización de una epistemología de carácter normativo (Foucault, 1969: 249-251).

Ahora bien, antes de dar paso al siguiente apartado, consideramos pertinente recapitular la estrategia de lectura propuesta. En ese sentido, cabe destacar que la arqueología foucaulteana de los saberes acerca de "lo humano", en lugar de contribuir a la realización de una epistemología normativa, permite llevar a cabo una crítica ontológico-política respecto de la actualidad. Interpretación 
que erigimos a partir de la puesta en juego de tres pares categoriales, dos introducidos por el propio Foucault y el tercero incorporado por quien escribe estas líneas. La articulación de los pares introducidos por Foucault permitió perfilar dos senderos para el trabajo filosófico que, declinado hacia la epistemología de las ciencias humanas, daría lugar a un tercer par. Tenemos, entonces, una filosofía analítica de la verdad en general, preocupada por las posibilidades del conocimiento y sus límites infranqueables, es decir por la elaboración de una teoría del conocimiento que, respecto de las ciencias humanas, permitiría perfilar una crítica epistemológica normativa acerca de su objetividad cognoscitiva. Frente a lo cual la propuesta foucaulteana de llevar a cabo una ontología de la actualidad se entronca con el abordaje arqueológico del saber y da lugar, respecto de las ciencias humanas, a una crítica epistemológica que -en lugar de bastarse a sí misma- se configura como un aporte para la problematización ontológico-política de la actualidad, por medio de la crítica de las formas de objetivación.

\section{La crítica política del saber como crítica de las formas de objetivación}

Foucault se propone mostrar cómo las prácticas sociales pueden llegar a engendrar dominios de saber que no sólo hacen que aparezcan nuevos objetos, conceptos y técnicas, sino que hacen nacer, además, formas totalmente nuevas de sujetos $y$ sujetos de conocimiento. En este sentido, Foucault afirma que el sujeto de conocimiento posee una historia, la relación del sujeto con el objeto, y, más claramente, la verdad misma tiene una historia 
En este apartado nos detendremos en la problematización foucaulteana del método genealógico. Específicamente, dirigiremos el foco de lectura hacia lo que denominamos como "ecos arqueológicos de la genealogía”, noción en la que consideramos que pueden distinguirse tres aristas: por un lado, el ya mencionado ejercicio de relectura del archivo de las ciencias humanas que Foucault realiza en el contexto de sus trabajos genealógicos; por otra parte, en tanto que la genealogía problematiza las relaciones de saber-poder (Foucault, 1975), no puede soslayarse la centralidad que la nociones de saber y práctica discursiva, forjadas en el seno de la arqueología, conservan en las genealogías elaboradas por Foucault en la década del setenta; por último, otro "eco arqueológico" que consideramos insoslayable, es la problematización de la genealogía como un método de trabajo filosófico que se despliega en las canteras de la historia, y que prescinde de adoptar una perspectiva crítica articulada por el par "teleología-normación", tal como lo explicitaremos a continuación.

Sin más preámbulos, en sintonía con la cita del especialista Marcelo Raffin que hemos colocado como epígrafe, nos remitimos -en primer lugar- a la "Leçons sur Nietzsche" dictada por Foucault en Montreal en 1971; lección en la que el filósofo problematiza, a partir de una lectura de Nietzsche, la posibilidad de trazar "una historia de la verdad que no se apoye en la verdad". Historia de la verdad a la que presenta en oposición a la elaborada dentro del marco de la filosofía positivista comteana:

En esta historia positivista, la verdad no está dada al comienzo. Durante mucho tiempo, el conocimiento la busca: ciego, titubeante. La verdad se da como el resultado de una historia. Pero esa relación finalmente establecida entre la verdad y el conocimiento es una relación de derecho que se plantea al comienzo. El conocimiento está hecho para ser conocimiento de la verdad. Hay una 
copertenencia de origen entre la verdad y el conocimiento. [...] El atrevimiento de Nietzsche consiste en haber desanudado esas implicaciones. Y haber dicho: la verdad sobreviene al conocimiento -sin que el conocimiento esté destinado a la verdad, sin que ella sea la esencia del conocer. [...] Pensar el conocimiento como un proceso histórico previo a toda problemática de la verdad, y más fundamentalmente que en la relación sujeto-objeto. El conocimiento liberado de la relación sujeto-objeto, es el saber (Foucault, 2011: 199-205).

Sostenemos, entonces, que la problematización genealógica recupera los desarrollos arqueológicos, puesto que la "des-implicación" entre conocimiento y verdad, que se liga a la posibilidad de repensar el conocimiento en términos de saber (es decir, de pensarlo por fuera de la relación sujeto-objeto), retoma la forma de problematización forjada por Foucault en el marco de la contraposición entre teoría del conocimiento y arqueología del saber. Cabe remarcar que, en la cita precedente, el filósofo vincula la noción de que el saber no se da entre sujeto y objeto, sino que ambos términos le son inmanentes, al hecho de que la interrogación se genera por fuera del problema de la verdad, valga la redundancia, por fuera de un problema pensable al interior de la relación cognoscitiva. De este modo, en su lectura de Nietzsche, Foucault destaca la posibilidad de llevar a cabo una historia de la verdad, en la que no entren en juego las verdades inmanentes a la formación discursiva de los saberes contemporáneos de modo normativo, tanto epistemológica como historiográficamente. En términos del propio Foucault:

Investigaciones realizadas previamente permitieron reconocer un nivel singular entre aquellos que permiten analizar los sistemas de pensamiento: el de las prácticas discursivas. Se trata de una sistema- 
ticidad que no es de tipo lógico ni lingüístico. Las prácticas discursivas se caracterizan por el recorte de un campo de objetos, la definición de una perspectiva legítima para el sujeto de conocimiento, la fijación de normas para la elaboración de conceptos y teorías. Cada una de ellas supone, entonces, un juego de prescripciones que rigen las exclusiones y elecciones. [...] Los caracteres generales de esas prácticas y los métodos propios para analizarlas se inventariaron bajo el nombre de arqueología (Foucault, 2011: 217-218).

El desplazamiento arqueológico del conocimiento hacia el saber habilita una forma de problematización que se erige por fuera de la problemática de la verdad y, por ende, queda al abrigo de valerse del "conocimiento actual" en términos historiográficamente teleológicos y epistemológicamente normativos, es decir de apoyarse en la verdad a la hora de indagar acerca de su historia. Por otra parte, en 1971 Michel Foucault publica "Nietzsche, la généalogie, l'histoire" (1994d), en el que -por medio de una lectura de Nietzsche- retoma la distinción entre Ursprung y Erfindung, términos alemanes que implican la noción de "origen" e "invención” respectivamente. En esta lectura, Ursprung es vinculado con la noción metafísica de "origen fuente", de "origen transhistórico", mientras Erfindung se liga a la problemática concreta de la procedencia (Herkunft) y de las condiciones de posibilidad para la emergencia o surgimiento (Entstehung) de las prácticas.

Dos años más tarde Foucault dictará, en Río de Janeiro, el ciclo de conferencias La vérité et les formes juridiques; en la primera de ellas, se detendría nuevamente en la exposición de su lectura de la genealogía nietzscheana. Allí, destacará que las herramientas nietzscheanas habilitan a la realización de una historia de la verdad descargada de un enfoque teleológico y normativo. En ese sentido, no podemos pasar por alto que al caracterizar el trabajo que llevaría a cabo a lo largo de las conferencias sostuvo que: 
Presentaré algunos esbozos de esta historia a partir de las prácticas judiciales de donde nacieron los modelos de verdad que circulan todavía en nuestra sociedad, que se imponen todavía y que valen no solamente en el dominio de la política, en el dominio del comportamiento cotidiano, sino hasta en el orden de la ciencia. Hasta en el orden de la ciencia se encuentran los modelos de verdad cuya formación continúan a las estructuras políticas que no se imponen desde el exterior al sujeto de conocimiento, sino que son, ellas mismas, constitutivas del sujeto de conocimiento (Foucault, 1994e: 553).

A partir de la lectura realizada a lo largo de las líneas precedentes, nos abocamos ahora a la revisión de la noción de crítica política del saber que Foucault presenta -en contraposición a la crítica de la ideología- en las clases del 10 y del 17 de enero de 1979, con las que diera inicio al curso Naissance de la biopolitique dictado en Collège de France. Allí, al explicitar las "cuestiones de método" que vertebran la problematización respecto de la formación de la economía política y el surgimiento del liberalismo como prisma reflexivo gubernamental, el filósofo planteó que:

La crítica que les propongo consiste en determinar bajo qué condiciones y con qué efectos se ejerce una veridicción, es decir, una vez más, un tipo de formulación dependiente de ciertas reglas de verificación y falseamiento. [...] No es la historia de lo verdadero, no es la historia de lo falso, es la historia de la veridicción la que posee importancia política (Foucault, 2004a: 37-38).

Frente a la puesta en cuestión del discurso científico decimonónico en términos de "conocimiento superado" por la ciencia actual, cuyo carácter político se ligaría con el hecho de que se trataba 
de "conocimiento todavía no suficientemente elaborado", "contaminado ideológicamente", que tendría por presunta función la "legitimación del poder", la crítica política del saber llevada a cabo por el genealogista permite dar cuenta del modo en que en determinado momento histórico se produjo un acoplamiento entre una serie de prácticas y un régimen de veridicción. Tal como se desprende de la palabra foucaulteana, la problematización de los saberes apuntará a dar cuenta del modo en que los mismos articularon una serie de prácticas, constituyendo ciertos objetos pasibles de ser interrogados a partir de determinadas reglas de verificación y falseamiento. Es decir, que de lo que se trata, a la hora de llevar a cabo una crítica política del saber, es ni más ni menos que de la realización de una crítica de las formas de objetivación inmanentes a determinado régimen de veridicción. Crítica epistemológica jalonada por objetivos ontológico-políticos que permite dar cuenta de la formación inmanente de los objetos y de las modalidades enunciativas que configuran las posiciones de sujeto; es decir, cómo se constituyen quién habla y de qué habla en el marco de determinado régimen de veridicción.

Enfatizamos, entonces, que la crítica política del saber posibilita la problematización del modo en que históricamente - de manera inmanente a cierta disposición epistémica- se constituyen objetos y posiciones de sujeto. Por lo tanto, en lugar de brindar herramientas para la realización de una crítica normativa respecto de la "objetividad cognoscitiva” de las ciencias humanas, la crítica política se desplaza hacia la puesta en cuestión de las formas de objetivación. Tal como Foucault lo hiciera de manera recurrente, por ejemplo respecto de la constitución de la delincuencia (Foucault, 1975), de la sexualidad y de la anormalidad (Foucault, 1976), del mercado, del homo œeconomicus y del capital humano (Foucault, 2004a; 2004b). Abordaje de los saberes acerca de "lo humano" que, en lugar de cuestionar su "falta de objetividad" y contraponer un "modo 
adecuando de conocer", se ejercita por medio de la problematización acerca de la formación de dichos objetos, desplegando así un conjunto de herramientas para la crítica epistemológica de las ciencias humanas que se encuentra jalonada por la preocupación ontológico-política respecto de la constitución de la actualidad. De este modo, en lugar de "la denuncia" del carácter ideológico, sesgado o pseudocientífico de dichos saberes, encontramos en la crítica foucaulteana una indagación acerca del modo en que la formación de dichas ciencias contribuye a la constitución de focos de experiencia, articulándose con el ejercicio del poder por medio de prácticas como el examen y la disciplina (Foucault, 1975), la regulación biopolítica (Foucault, 1976) y la gubernamentalidad liberal y neoliberal (Foucault, 2004a; 2004b).

\section{Palabras finales}

La arqueología como método, y especificamente Las palabras y las cosas, son una propedéutica a la genealogía. La genealogía, tal como nos fue presentada por Foucault, no es la crisis de la arqueología, sino que ellas se apuntalan mutuamente.

Defert, (2011: 271)

A lo largo del presente artículo, al tomar como punto de apoyo el característico gesto foucaulteano de recuperación y reelaboración recurrente de sus trabajos, hemos revisado el archivo Foucault para rastrear series documentales que nos permitan releer la propuesta foucaulteana de llevar a cabo una crítica política del saber. En ese sentido, en primer lugar, nos detuvimos en el abordaje recurrente del discurso de las ciencias humanas dentro del marco de problematización de la filosofía como actividad de diagnóstico respecto 
de la actualidad; luego, pusimos el foco de lectura en el tipo de crítica de los saberes acerca de "lo humano" que Foucault configura en el seno de su problematización arqueológica del saber; tras lo cual, por medio de la explicitación de lo que hemos denominado como "ecos arqueológicos de la genealogía", dirigimos nuestro abordaje de los documentos en pos de revisar la noción de crítica política del saber.

A partir de la lectura realizada, sostenemos entonces que nos encontramos frente a dos inflexiones posibles para la crítica epistemológica de las ciencias humanas, el camino de la "analítica de la verdad" y la teoría del conocimiento, que da lugar al "perenne problema" de la "objetividad cognoscitiva" de dichas ciencias; inflexión frente a la que el discurso foucaulteano introduce un desplazamiento del eje de problematización, perfilando una crítica epistemológica y ontológico-política de las formas de objetivación inmanentes a la formación de los saberes. Así, en lugar de cuestionar epistemológicamente el discurso de las mencionadas ciencias en función de su supuesta "falta de objetividad", y de oponerles una forma "más adecuada" de abordar sus objetos, de lo que se trata es de problematizar el modo en que sus formas de objetivación contribuyen a la constitución ontológico-política de la actualidad. Tarea respecto de la que el filósofo introdujo la noción de crítica política del saber, a la que hemos caracterizado como una crítica de las formas de objetivación inmanentes a la formación del discurso de las ciencias humanas. Forma de objetivación cuya contracara no es ni más ni menos que la constitución inmanente de modalidades enunciativas, es decir del recorte de posiciones de sujeto. Constitución del par sujeto-objeto en torno al que se configura, entonces, un discurso atravesado por la contraposición entre lo verdadero y lo falso. 


\section{Bibliografía}

Brossat, Alain, 2013, "L'archive et les archives. Archéologie des discours et gouvernement des vivants", en Materiali Foucaultiani, vol. 2, núm. 4.

Castro, Edgardo, 1995, Pensar a Foucault: interrogantes filosóficos de La arqueología del saber, Editorial Biblos, Buenos Aires.

, 2011, Lecturas foucaulteanas. Una historia conceptual de la biopolitica, UnIPE/Editorial Universitaria, Buenos Aires.

Castro-Gómez, Santiago, 2010 Historia de la gubernamentalidad. Razón de Estado, liberalismo y neoliberalismo en Michel Foucault, Siglo del Hombre Editores, Bogotá.

Defert, Daniel, 2011, "Situation du Cours", en Michel Foucault, Leçons sur la volonté de savoir. Cours au Collège de France. 19701971, Michel Foucault, Éditions Gallimard seuIL, París.

Fontana, Alessandro, Bertani, Mauro, 1997, "Situation du Cours", en Michel Foucault, "Il faut défendre la société". Cours au Collège de France. 1976, Éditions Gallimard seuiL, París.

Foucault, Michel, 1963, Naissance de la clinique. Une archéologie du regard médical, París: Presses Universitaires de France, París.

, 1966, Les mots et les choses. Une archéologie des sciences humaines, Gallimard, París.

, 1969, L'archéologie du savoir, Éditions Gallimard, París.

, 1972, Histoire de la folie à l'âge classique, Éditions Gallimard, París.

, 1975, Surveiller et punir. Naissance de la prison, Éditions Gallimard, París.

, 1976, Histoire de la sexualité 1. La volonté de savoir, Éditions Gallimard, París. 
, 1994a, "Qu`est-ce qu’un philosophe?", en Dits et écrits. 1954-1988. I. 1954-1969, Éditions Gallimard, París.

, 1994b, "La philosophie structuraliste permet de diagnostiquer ce qu'est 'aujourd'hui'”, en Dits et écrits. 1954-1988. I. 1954-1969, Éditions Gallimard, París.

, 1994c, "Sur l’archéologie des sciences. Réponse au Cercle d'épistémologie”, en Dits et écrits. 1954-1988. I. 1954-1969, Éditions Gallimard, París.

, 1994d, "Nietzsche, la généalogie, l'histoire", en Dits et écrits. 1954-1988. II. 1970-1975, Éditions Gallimard, París.

, 1994e, "La vérité et les formes juridiques", en Dits et écrits. 1954-1988. II. 1970-1975, Éditions Gallimard, París.

, 2004a, Naissance de la biopolitique. Cours au Collège de France. 1978-1979, Éditions Gallimard seuil, París.

,2004b, Sécurité, Territoire, Population. Cours au Collège de France, Éditions Gallimard sEuIL, París.

, 2008, Le gouvernement de soi et des autres. Cours au Collège de France. 1982-1983, Éditions Gallimard seuil, París.

, 2011, Leçons sur la volonté de savoir. Cours au Collège de France, 1970-1971, Éditions Gallimard sEuIL, París.

Gutting, Gary, 1989, Michel Foucault's archaeology of scientific reason, Cambridge University Press, Cambridge.

Lehm, Vanessa y Miguel Vatter, 2014, "Introduction”, en Vanessa Lehm y Miguel Vatter (eds.), The Government of Life. Foucault, Biopolitcs and Neoliberalism, Fordham University Press, Nueva York.

Mascaretti, Giovanni Maria, 2014, "Michel Foucault on Problematization, Parrhesia and Critique", Materiali Foucaultiani, año 3, núm. 5-6. 
Muhle, Maria, 2012, "Histoire(s) de la vie de Canguilhem a Foucault", en AA.VV., Epistemology and History. From Bachelard and Canguilhem to Today's History of Science, Max Planck Institut for the History of Science.

Paltrinieri, Luca, 2014, "Archeologia della volontà. Una preistoria delle Lezioni sulla volontà di sapere", Quadranti. Rivista Internazionale di Filosofia Contemporanea, vol. 2, núm. 1.

Raffin, Marcelo, 2014, "Natureza e política: uma tensão no pensamento de Michel Foucault", Ágora Filosófica, vol.1, núm. 2.

, 2015, "La verdad y las formas políticas: la lectura temprana de la tragedia de Edipo en Michel Foucault", Anacronismo e Irrupción. Revista de Teoría y Filosofía Politica Clásica y Moderna, vol. 5, núm. 8 .

Wallenstein, Sven-Olov, 2013, "Introduction: Foucault, Biopolitics, and Governmentality" en Jakob Nilsson y Sven-Olov Wallenstein (eds.), Foucault, Biopolitics and Governmentality, Södertörn, Södertörn University the Library, Estocolmo. 